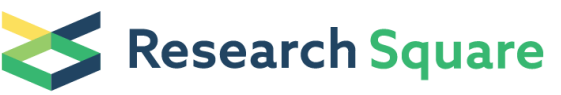

Preprints are preliminary reports that have not undergone peer review. They should not be considered conclusive, used to inform clinical practice, or referenced by the media as validated information.

\section{Correlation Network Analyses Based on Metagenomics and Multi-type Metabolomic Data Identified Biomarkers of Coronary Artery Disease}

\section{Chaoran Dong}

Chinese Academy of Medical Sciences \& Peking Union Medical College Institute of Materia Medica https://orcid.org/0000-0003-0424-9746

\section{Zilong He}

Institute of Microbiology Chinese Academy of Sciences

Qianhui Zhu

Institute of Microbiology Chinese Academy of Sciences

Jianxun Liu

Institute of Microbiology Chinese Academy of Sciences

\section{Feng Gao}

Chinese Academy of Medical Sciences \& Peking Union Medical College Institute of Materia Medica

\section{Kexin Li}

Institute of Microbiology Chinese Academy of Sciences

\section{Shanshan Sun}

Chinese Academy of Medical Sciences \& Peking Union Medical College Institute of Materia Medica Qifeng Liu

Chinese Academy of Medical Sciences \& Peking Union Medical College Institute of Materia Medica

\section{Yinghong Wang}

Chinese Academy of Medical Sciences \& Peking Union Medical College Institute of Materia Medica

\section{Yida Tang}

Chinese Academy of Medical Sciences \& Peking Union Medical College Fuwai Hospital

\section{Songnian $\mathrm{Hu}$}

Institute of Microbiology Chinese Academy of Sciences

Haibo Zhu ( $\nabla$ zhuhaibo@imm.ac.cn )

Chinese Academy of Medical Sciences \& Peking Union Medical College Institute of Materia Medica

\section{Research article}

Keywords: coronary artery disease, metagenomics, metabolomics, correlation network, biomarkers

Posted Date: September 4th, 2020 
DOI: https://doi.org/10.21203/rs.3.rs-65514/v1

License: (c) (i) This work is licensed under a Creative Commons Attribution 4.0 International License. Read Full License 


\section{Abstract}

Background: Coronary artery disease (CAD) is a complex, multifactorial disease and the underlying pathogenesis is unclear. It is essential to improve our understanding of the aetiology and pathogenesis of CAD for developing effective methods of early diagnosis and treatment.

Results: We recruited 190 participants including normal coronary artery $(n=49)$, stable coronary artery disease $(n=93)$ and acute myocardial infarction patients $(n=48)$. We combined metagenomics (16S rRNA sequencing and gut microbiome whole-genome sequencing) and multi-type metabolomics (serum, faeces and urine) analyses to determine the correlations among metabolites, the microbiota and clinical indexes to identify biomarkers of CAD. Compared with the faecal metabolites, serum and urine metabolites exhibited strong correlation with clinical indexes. Comparing the three types of metabolome, we discovered that the faecal and urine metabolome was more suitable than the serum metabolome for correlation analysis with the microbiota. Furthermore, we found that the serum and urine metabolome was more suitable than the faecal metabolome for correlation analysis with the clinical indexes. We constructed, for the first time, the relationship networks among the microbiota, metabolites and pathways. Through the relationship network analysis, we identified some important differential metabolites and delineated how these metabolites are related to the differentially abundant microbes.

Conclusions: In our research, we used metagenome and multi-type metabolome profiling based on hundreds of samples to figure out the correlations among metabolites, microbiota and clinical indexes. In addition, we firstly constructed the relationship networks of microbiota, metabolites and pathways. We believe our findings can help the researchers to further understand the pathogenesis of CAD.

\section{Background}

Coronary artery disease (CAD) is a heart disease caused by the accumulation of atherosclerotic plaques in blood vessels[1]. Based on clinical symptoms, the extent of arterial blockage and the degree of myocardial injury, CAD is divided into different categories, including stable coronary artery disease (SCAD) and acute myocardial infarction (AMI)[2]. Stable coronary artery disease (SCAD) refers to the syndrome of recurrent, transient episodes of chest pain reflecting demand-supply mismatch, that is, angina pectoris[3]. AMI is characterized by acute myocardial ischaemia and the elevated cardiac troponin values (cTn) with at least one value above the 99th percentile upper reference limit (URL)[4]. Epidemiological studies of CAD have shown that some clinical indexes such as age, male gender and smoking can increase the risk of myocardial infarction. Simultaneously, low-density lipoprotein cholesterol (LDL-C), triglyceride-rich lipoproteins, or high-density lipoprotein cholesterol (HDL-C) are associated with $C A D$ [1]. Although Invasive coronary angiography $(C A)$ is the gold standard for diagnosis of coronary artery disease (CAD), it is so invasive that it can cause various complications[5]. Improving our understanding of CAD aetiology and pathogenesis is essential to developing effective methods for early diagnosis and treatment. 
Recent studies have shown an association between the gut microbiome and cardiovascular diseases[6]. Several metagenome-wide association studies have revealed that the composition of the human intestinal microbiome can shape human health, and that specific gut microbes are associated with many diseases[7-9]. Many studies have proven that the composition of the gut microbiome is influenced by the diet, lifestyle and genetic susceptibility of the host[10-12]. Researches show that the disordered human gut microbiome can lead to diseases, such as cardiovascular disease, metabolic syndrome and type 2 diabetes $[7,10,13]$.

In terms of the contribution of the microbiome to cardiovascular diseases, several breakthroughs have been made by performing 16rRNA-seq or metagenome sequencing. For example, Li et al. performed comprehensive metagenomic and metabolomic analyses, described a novel causal role of aberrant gut microbiota in hypertension pathogenesis and also verified the results in mouse models[14]. Liu et al. identified 29 metabolite modules that were correlated with CAD phenotypes and constructed a classifier that could discriminate cases from controls[6]. Zhou et al. provided the first evidence that cardiovascular outcomes following myocardial infarction (MI) are driven by the intestinal microbiota[15].

Despite these advances, no one has compared the differential correlations between the gut microbiota/clinical indexes and three key metabolome profiles: serum, faeces and urine metabolomes. In addition, few studies have described the relationships among metabolites, the gut microbiota and pathways. To better understand the pathogenesis of CAD, we analyzed metagenome (16S rRNA sequencing and gut microbiome whole-genome sequencing) and multi-type metabolome (serum, faeces and urine) data to determine the correlations among metabolites, the microbiota and clinical indexes, and to identify biomarkers of CAD in 190 participants. Additionally, we systematically compared the results for three types of metabolome (serum, faeces and urine). We hope our findings will help the researchers to understand the pathogenesis of CAD much better.

\section{Results}

\subsection{Clinical characteristics of CAD groups}

We collected 190 participants including 49 individuals with a normal coronary artery (NCA) profile, 93 patients with stable coronary artery disease (SCAD) and 48 patients with acute myocardial infarction (AMI) from Fuwai Hospital (Beijing, China) (Table 1). In terms of patient demographics, we found that sex was significantly associated with the three groups $(p<0.05)$, while smoking and drinking were only significantly different in NCA vs. SCAD and NCA vs. AMI $(p<0.05)$. Only hypertension was significantly different in NCA vs. SCAD and SCAD vs. AMI. In laboratory parameters, C-reactive protein (CRP) levels was significantly different among the three groups $(p<0.05)$, LDL cholesterol (LDL-C) and total cholesterol (TC) levels were significantly different in NCA vs. SCAD and NCA vs. AMI $(p<0.05)$. In terms of the Gini coefficients of all clinical indexes (Figure S2), lactic acid dehydrogenase (LDH), aspartate transaminase (AST), CRP, LDL-C, TC and creatine kinase-MB (CK-MB) were the top ranked, demonstrating that these indexes have auxiliary significance for the clinical diagnosis of CAD. 
Table 1

Summary of clinical indexes

\begin{tabular}{|c|c|c|c|c|c|c|}
\hline & $\operatorname{NCA}(n=49)$ & $s C A D(n=93)$ & AMI $(n=48)$ & $\begin{array}{l}p \\
\text { value } \\
\text { (NCA } \\
\text { vs. } \\
\text { sCAD) }\end{array}$ & $\begin{array}{l}p \\
\text { value } \\
\text { (NCA } \\
\text { vs. } \\
\text { AMI) }\end{array}$ & $\begin{array}{l}p \\
\text { value } \\
\text { (sCAD } \\
\text { vs. } \\
\text { AMI) }\end{array}$ \\
\hline \multicolumn{7}{|l|}{ Demographics } \\
\hline $\begin{array}{l}\text { Sex } \\
\text { (Male/Female) }\end{array}$ & $19 / 29$ & $71 / 22$ & $46 / 3$ & $<0.001$ & $<.001$ & 0.010 \\
\hline Age & $57.06 \pm 9.23$ & $58.43 \pm 9.26$ & $58.04 \pm 13.21$ & 0.428 & 0.639 & 0.950 \\
\hline Smoking, case(\%) & $12(25.00)$ & $61(65.59)$ & $32(65.31)$ & $<.001$ & $<.001$ & 1.000 \\
\hline Drinking, case(\%) & 9 (18.75) & $42(45.16)$ & $26(53.06)$ & 0.002 & $<.001$ & 0.480 \\
\hline \multicolumn{7}{|l|}{ Medical history } \\
\hline $\begin{array}{l}\text { Hypertension, } \\
\text { case(\%) }\end{array}$ & $20(41.67)$ & 68 (73.12) & $25(51.02)$ & $\begin{array}{l}< \\
0.001\end{array}$ & 0.418 & 0.017 \\
\hline $\begin{array}{l}\text { Type } 2 \text { diabetes, } \\
\text { case }(\%)\end{array}$ & $13(27.08)$ & $32(34.41)$ & $18(36.73)$ & 0.566 & 0.385 & 0.710 \\
\hline $\begin{array}{l}\text { Hyperlipidemia, } \\
\text { case(\%) }\end{array}$ & $31(64.58)$ & $63(67.74)$ & 35 (71.43) & 0.710 & 0.518 & 0.706 \\
\hline Blood glucose & $7.25 \pm 3.61$ & $6.38 \pm 1.81$ & $6.74 \pm 2.02$ & 0.534 & 0.735 & 0.302 \\
\hline \multicolumn{7}{|l|}{$\begin{array}{l}\text { Laboratory } \\
\text { parameters }\end{array}$} \\
\hline PN & $59.34 \pm 11.54$ & $65.49 \pm 8.18$ & $79.58 \pm 99.06$ & $\begin{array}{l}<.001 \\
0.00\end{array}$ & $<.001$ & 0.828 \\
\hline ANC & $3.85 \pm 1.51$ & $4.72 \pm 1.67$ & $6.27 \pm 9.95$ & $<0.001$ & $<.001$ & 0.537 \\
\hline PL & $31.2 \pm 10.18$ & $25.46 \pm 7.21$ & $24.65 \pm 6.77$ & $<0.001$ & $\begin{array}{l}< \\
0.001\end{array}$ & 0.629 \\
\hline ALC & $1.99 \pm 0.77$ & $1.77 \pm 0.62$ & $1.8 \pm 0.6$ & 0.093 & 0.135 & 0.988 \\
\hline PM & $6.38 \pm 1.72$ & $6.62 \pm 2.17$ & $7.27 \pm 2.13$ & 0.519 & 0.017 & 0.037 \\
\hline AMC & $0.39 \pm 0.11$ & $0.59 \pm 0.9$ & $0.53 \pm 0.18$ & 0.010 & $<.001$ & 0.053 \\
\hline RBC & $4.45 \pm 0.51$ & $4.57 \pm 0.82$ & $4.53 \pm 0.5$ & 0.036 & 0.350 & 0.320 \\
\hline PLT & $219.46 \pm 57.39$ & $227.44 \pm 71.95$ & $234.59 \pm 69.06$ & 0.848 & 0.444 & 0.517 \\
\hline
\end{tabular}




\begin{tabular}{|c|c|c|c|c|c|c|}
\hline D-dimer & $0.46 \pm 0.48$ & $0.67 \pm 1.52$ & $0.75 \pm 1.13$ & 0.776 & 0.581 & 0.350 \\
\hline FDP & $2.47 \pm 0.68$ & $2.79 \pm 2.34$ & $3.12 \pm 1.55$ & 0.981 & 0.071 & 0.020 \\
\hline BMI & $24.82 \pm 3.6$ & $25.55 \pm 2.83$ & $26.41 \pm 3.4$ & 0.124 & 0.043 & 0.219 \\
\hline $\mathrm{HbA1c}$ & $6.43 \pm 1.59$ & $9.12 \pm 20.62$ & $9.51 \pm 20.84$ & 0.618 & 0.699 & 0.299 \\
\hline TC & $4.78 \pm 1.18$ & $4.12 \pm 1.98$ & $3.83 \pm 0.84$ & $\begin{array}{l}< \\
0.001\end{array}$ & $\begin{array}{l}< \\
0.001\end{array}$ & 0.797 \\
\hline HDL & $1.35 \pm 0.41$ & $1.16 \pm 0.46$ & $1.06 \pm 0.3$ & $\begin{array}{l}<.001 \\
0.00\end{array}$ & $\begin{array}{l}<.001 \\
0.00\end{array}$ & 0.189 \\
\hline Triglycerides & $1.8 \pm 1.02$ & $1.81 \pm 1.01$ & $1.45 \pm 0.98$ & 0.900 & 0.047 & 0.010 \\
\hline LDL-C & $3.09 \pm 1.07$ & $2.38 \pm 0.91$ & $2.2 \pm 0.77$ & $\begin{array}{l}<.001 \\
0\end{array}$ & $\begin{array}{l}< \\
0.001\end{array}$ & 0.459 \\
\hline CRP & $1.24 \pm 1.11$ & $3.79 \pm 6.05$ & $9.32 \pm 14.61$ & $<.001$ & $<.001$ & $\begin{array}{l}< \\
0.001\end{array}$ \\
\hline Creatinine & $71.85 \pm 10.1$ & $82.14 \pm 20.51$ & $77.88 \pm 15.52$ & $\begin{array}{l}<.001 \\
0\end{array}$ & 0.032 & 0.241 \\
\hline WBC & $7.48 \pm 8.31$ & $7.33 \pm 2.62$ & $7.31 \pm 2.05$ & 0.022 & 0.004 & 0.952 \\
\hline ALT & $24.76 \pm 14.27$ & $31.95 \pm 31.69$ & $36.45 \pm 21.2$ & 0.129 & $\begin{array}{l}< \\
0.001\end{array}$ & 0.013 \\
\hline AST & $22.96 \pm 16.99$ & $23.41 \pm 21.95$ & $61.24 \pm 69.14$ & 0.231 & $<.001$ & $<.001$ \\
\hline CK-MB & $11.83 \pm 3.06$ & $19.54 \pm 41.39$ & $32.63 \pm 47.89$ & 0.617 & 0.214 & 0.425 \\
\hline CK & $83.17 \pm 40.51$ & $113.72 \pm 183.55$ & $419.63 \pm 563.23$ & 0.927 & $<.001$ & $\begin{array}{l}< \\
0.001\end{array}$ \\
\hline LDH & $171.58 \pm 44.5$ & $176.94 \pm 44.49$ & $316.63 \pm 228.12$ & 0.573 & $\begin{array}{l}< \\
0.001\end{array}$ & $\begin{array}{l}< \\
0.001\end{array}$ \\
\hline FT3 & $2.92 \pm 0.27$ & $2.92 \pm 0.3$ & $2.62 \pm 0.55$ & 0.766 & $<.001$ & $\begin{array}{l}< \\
0.001\end{array}$ \\
\hline FT4 & $1.09 \pm 0.18$ & $1.15 \pm 0.32$ & $1.08 \pm 0.42$ & 0.676 & 0.018 & 0.003 \\
\hline T3 & $1.08 \pm 0.16$ & $1.15 \pm 0.39$ & $1.01 \pm 0.38$ & 0.863 & 0.007 & 0.002 \\
\hline T4 & $7.86 \pm 1.8$ & $7.74 \pm 1.86$ & $7.43 \pm 1.95$ & 0.789 & 0.442 & 0.409 \\
\hline TSH & $4.18 \pm 12.35$ & $2.62 \pm 2.82$ & $1.76 \pm 1.28$ & 0.481 & 0.098 & 0.009 \\
\hline
\end{tabular}




\subsection{Identification of differential microbiotas in the gut microbiome between the CAD subgroups}

We first aimed to explore the changes in intestinal microbial community composition in the three CAD subgroups. We used QIIME2 and LEfSe to analyze the 16S rRNA sequencing data from the 190 participants[20]. The alpha diversity plot (Figure S3) showed that the sequencing data from all the samples was saturated, and the richness was diverse. We then used the cPCoA method to reveal the beta diversity of microbial composition among the CAD subgroups (Figure S4). In addition, the taxonomic classification at the phylum level of the CAD subgroups is shown in Figure S5. Here, we found that the 190 samples were mainly composed of the phyla Bacteroidetes and Firmicutes.

We focused next on the microbes with significant differential abundances among the three CAD subgroups (LDA > 2) (Figure 1). We identified 25 different taxa between the NCA and sCAD groups (Figure 1A). For example, Bacteroides and Escherichia were found at a higher abundance in the NCA group (LDA > 3), while Desulfovibrio and Parabacteroides were found at a higher abundance in the sCAD group (LDA $>2$ ). We also identified 34 significantly different taxa between the NCA and AMI groups (Figure 1B). Here, Desulfovibrio, Streptococcus, and Lacobacillus had higher abundances in the AMI group (LDA > 2), while Clostridium had a higher abundance in the NCA group (LDA > 3). Finally, we found 69 significantly different taxa between the SCAD and AMI groups (Figure 1C). Notably, Streptococcus, Alistipes, Olsenella, Actinomyces, and Escherichia had higher abundances in the AMI group (LDA > 2), and Prevotella, Clostridium, and Lactobacillus had higher abundances in the sCAD group (LDA > 3). Together, we have identified taxa with significantly different abundances in different CAD subgroups.

\subsection{Identification of differential metabolites in multi-type metabolomes between the CAD subgroups}

We next used NMR (Nuclear Magnetic Resonance) to detect metabolites in the three types of metabolome (faeces, serum and urine) in samples from NCA, sCAD and AMI participants. We detected total of 29, 33 and 31 metabolites in the faeses, serum and urine, respectively. In NCA vs. sCAD, we identified 3, 18 and 18 metabolites that were differential detectable in the faeces, serum and urine, respectively (Table 2$)(p$ $<0.1)$. The methanol level was also significantly different in the three types of metabolome, and the levels of other four metabolites, including creatinine, were significantly different in the serum and urine metabolome. In SCAD vs. AMI, we detected 7, 21 and 18 differentially detectable metabolites in the faeces, serum and urine, respectively (Table 2$)(p<0.1)$. Similar to NCA vs. SCAD, the levels of five metabolites, including creatinine, were significantly different in the serum and urine metabolomes. Finally, in NCA vs. AMI, we detected 6, 18 and 14 differentially detectable metabolites in the faeces, serum and urine, respectively (Table 2$)(p<0.1)$. Again, the levels of creatinine and alanine were significantly different in the serum and urine metabolome. Overall, we found that citrate and creatinine levels in the serum and 


\subsection{Integrated analyses reveal the relationships between the gut microbiota and differential faecal metabolites in the CAD subgroups}

To identify the relationships among the microbiota, metabolites and metabolic pathways, we performed an integrated analysis based on 16S rRNA gene, metagenomic, and metabolomics (faecal) sequencing data. 20 samples of each CAD subgroups were randomly selected for metagenomic sequencing. We identified the relationships between metabolites and metabolic pathways in the MetaCyc database[25] and obtained the relative abundances of taxa and metabolic pathways by analyzing metagenomic data. Then, we constructed relationship networks based on the metabolic pathways, differential metabolites and differentially abundant genera.

In the NCA vs. SCAD network (Figure 2), the differential abundant metabolite alanine and differentially abundant genus Escherichia/Streptococcus were linked through the metabolic pathways: L-alanine biosynthesis. In the SCAD vs. AMI network, we observed 6 differential metabolites, 13 metabolic pathways, and 12 differentially abundant genera. According to the previous studies, methionine and acetate are associated with the development of CAD [27-30]. And in our research, methionine and acetate were connected to differentially abundant genera, such as Escherichia, Streptococcus and Coprococcus through the L-methionine cycle I, pyruvate fermentation to acetate and lactate II metabolic pathways. Notably, we found that Escherichia was present in both the NCA vs. SCAD and SCAD vs. AMI networks. Finally, the NCA vs. AMI network contained 3 differential metabolites, 11 metabolic pathways and 6 genera. Interestingly, methionine and acetate were present in both the SCAD vs. AMI and NCA vs. AMI networks. With the exception of Desulfovibrio, the five other differentially abundant genera also existed in the SCAD vs. AMI network. These metabolites and genera might, therefore be associated with AMI occurrence. 
Table 2A

Differential metabolites in fecal samples among NCA, SCAD and AMI

\begin{tabular}{|lll|}
\hline Feces Metabolites & Fold Change & $p$ Value \\
\hline sCAD vs NCA & & \\
\hline Hypoxanthine & 1.198 & 0.0307 \\
\hline Methanol & 0.654 & 0.0887 \\
\hline Alanine & 1.126 & 0.0963 \\
\hline AMI vs NCA & & \\
\hline 1,3-Dihydroxyacetone & 0.841 & 0.0685 \\
\hline Acetate & 0.852 & 0.0835 \\
\hline Glucose & 0.794 & 0.0980 \\
\hline Malonate & 0.811 & 0.0506 \\
\hline Methionine & 1.183 & 0.0473 \\
\hline Phenylacetate & 1.315 & 0.0153 \\
\hline AMl vs sCAD & & \\
\hline 1,3-Dihydroxyacetone & 0.884 & 0.0686 \\
\hline Acetate & 0.801 & 0.0035 \\
\hline Alanine & 0.872 & 0.0702 \\
\hline Citrulline & 0.875 & 0.0538 \\
\hline Methionine & 1.165 & 0.0286 \\
\hline Phenylacetate & 1.323 & 0.0022 \\
\hline Tyrosine & 0.856 & 0.0605 \\
\hline
\end{tabular}


Table 2B

Differential metabolites in urine samples among NCA, SCAD and AMI

\begin{tabular}{|lll|}
\hline Urine Metabolites & Fold Change & $\boldsymbol{p}$ Value \\
\hline sCAD vs NCA & & \\
\hline 2-Hydroxyisobutyrate & 1.143 & 0.0268 \\
\hline 3-Hydroxyisovalerate & 0.858 & 0.0203 \\
\hline Betaine & 1.548 & 0.0265 \\
\hline Citrate & 0.779 & 0.0049 \\
\hline Creatinine & 1.275 & 0.0001 \\
\hline Galactose & 0.004 & $<0.0001$ \\
\hline Glycine & 1.131 & 0.0419 \\
\hline Glycolate & 1.128 & 0.0459 \\
\hline Isoleucine & 0.559 & $<0.0001$ \\
\hline Methanol & 0.821 & 0.0073 \\
\hline Methylsuccinate & 0.599 & 0.0071 \\
\hline N-Acetylglutamate & 0.811 & 0.0044 \\
\hline Phenylalanine & 1.428 & 0.0131 \\
\hline Tartrate & 0.627 & $<0.0001$ \\
\hline Taurine & 1.210 & 0.0863 \\
\hline Threonine & 1.125 & 0.0634 \\
\hline Tyrosine & 0.785 & 0.0164 \\
\hline Valine & 0.397 & $<0.0001$ \\
\hline AMl vs NCA & 0.840 & 0.0209 \\
\hline 2-Oxoglutarate & 0.650 & 0.0002 \\
\hline 3-Hydroxyisovalerate & 0.836 & 0.0221 \\
\hline Acetate & 4.041 & 0.0016 \\
\hline Alanine & 0.0001 \\
\hline Choline & & \\
\hline Citrate & 0.534 & \\
\hline
\end{tabular}




\begin{tabular}{|lll|}
\hline Creatinine & 0.805 & 0.0107 \\
\hline Glycolate & 0.777 & 0.0007 \\
\hline Hippurate & 0.487 & 0.0004 \\
\hline Isoleucine & 0.796 & 0.0364 \\
\hline Methanol & 0.791 & 0.0190 \\
\hline Pyruvate & 0.825 & 0.0170 \\
\hline Taurine & 1.413 & 0.0078 \\
\hline Trigonelline & 0.178 & 0.0542 \\
\hline AMl vs sCAD & & \\
\hline 2-Hydroxyisobutyrate & 0.829 & 0.0113 \\
\hline Acetate & 4.041 & $<0.0001$ \\
\hline Alanine & 0.766 & 0.0005 \\
\hline Betaine & 0.491 & 0.0013 \\
\hline Choline & 0.746 & 0.0004 \\
\hline Citrate & 1.834 & 0.0091 \\
\hline Creatinine & 0.686 & 0.0007 \\
\hline Formate & 0.631 & $<0.0001$ \\
\hline Galactose & 1.769 & 0.0137 \\
\hline Glycolate & 187.176 & $<0.0001$ \\
\hline Hippurate & 0.689 & $<0.0001$ \\
\hline Hypoxanthine & 0.797 & 0.0009 \\
\hline Isoleucine & 1.425 & 0.0855 \\
\hline N-Acetylglutamate & 1.148 & 0.0950 \\
\hline Tartrate & 0.0072 \\
\hline Threonine & 0.0260 \\
\hline Tyrosine & & \\
\hline Valine & & \\
\hline
\end{tabular}


Table 2C

Differential metabolites in serum samples among NCA, SCAD and AMI

\begin{tabular}{|lll|}
\hline Serum Metabolites & Fold Change & $p$ Value \\
\hline sCAD vs NCA & & \\
\hline Tyrosine & 0.915 & 0.0176 \\
\hline Proline & 1.104 & 0.0087 \\
\hline Methionine & 1.089 & 0.0124 \\
\hline Methanol & 0.630 & $<0.0001$ \\
\hline Isobutyrate & 1.186 & $<0.0001$ \\
\hline Glycine & 0.962 & 0.0667 \\
\hline Glycerol & 1.825 & $<0.0001$ \\
\hline Formate & 1.848 & 0.0026 \\
\hline Creatinine & 1.210 & $<0.0001$ \\
\hline Citrate & 0.933 & 0.0261 \\
\hline Alanine & 0.917 & 0.0113 \\
\hline 3-Hydroxybutyrate & 1.355 & 0.0577 \\
\hline 2-Hydroxybutyrate & 1.208 & $<0.0001$ \\
\hline VLDL/LDL & 0.909 & 0.0008 \\
\hline Ptdcho & 0.890 & 0.0007 \\
\hline N-Ac & 0.949 & 0.0002 \\
\hline lipid-CH ${ }_{2}$ CO & 0.950 & 0.0020 \\
\hline lipid-CH ${ }_{2}$ CH ${ }_{2}$ CO & 0.925 & $<0.0001$ \\
\hline AMl vs NCA & & 0.0867 \\
\hline Propylene glycol & 0.844 & $<0.0001$ \\
\hline Phenylalanine & 1.093 & 0.0374 \\
\hline Lactate & 1.263 & $<0.0001$ \\
\hline Isoleucine & 0.875 & 0.0037 \\
\hline Isobutyrate & 0.902 & 0.086 \\
\hline
\end{tabular}




\begin{tabular}{|lll|} 
Glycine & 0.900 & $<0.0001$ \\
\hline Glycerol & 1.479 & 0.0420 \\
\hline Dormate & 1.648 & 0.0840 \\
\hline Creatinine & 1.115 & 0.0341 \\
\hline Citrate & 0.872 & 0.0032 \\
\hline Betaine & 0.848 & 0.0026 \\
\hline Alanine & 0.852 & 0.0007 \\
\hline Acetone & 1.403 & 0.0166 \\
\hline 3-Hydroxybutyrate & 1.811 & 0.0062 \\
\hline 2-Hydroxybutyrate & 1.148 & 0.0381 \\
\hline lipid-CH=CH & 0.929 & 0.0513 \\
\hline VLDL/LDL & 0.914 & 0.0165 \\
\hline AMl vs sCAD & & 0.0041 \\
\hline Propylene glycol & 0.875 & 0.0001 \\
\hline Proline & 0.865 & 0.0004 \\
\hline Phenylalanine & 1.107 & 0.0013 \\
\hline Methionine & 0.902 & 0.0019 \\
\hline Methanol & 1.299 & 0.0230 \\
\hline Lactate & 1.289 & $<0.0001$ \\
\hline Isoleucine & 0.848 & $<0.0001$ \\
\hline Isobutyrate & 0.761 & $<0.0001$ \\
\hline Glycine & 0.935 & 0.0024 \\
\hline Glycerol & 0.810 & 0.0757 \\
\hline Dimethyl sulfone & 0.864 & 0.0141 \\
\hline Creatinine & 0.921 & 0.0810 \\
\hline Citrate & 0.934 & 0.0578 \\
\hline Blanine & 0.829 & \\
\hline
\end{tabular}




\begin{tabular}{|lcc|}
\hline 3-Hydroxybutyrate & 1.336 & 0.0860 \\
\hline N-Ac & 1.060 & 0.0844 \\
\hline lipid-CH2CO & 1.062 & $<0.0001$ \\
\hline lipid-CH2CH2CO & 1.032 & 0.0899 \\
\hline lipid-CH=CH & 1.064 & $<0.0001$ \\
\hline
\end{tabular}

\subsection{Correlation analysis between the gut microbiota and CAD clinical indexes}

To uncover the relationships between the gut microbiota and clinical indexes, we performed a spearman correlation analysis of the relative abundance of the gut microbiota with the clinical indexes of all samples (Figure 3). As the figure shows, a lots of significant correlations were found, for example, AST was significantly positively correlated with S.agalactiae, Streptococcus, and Streptococcaceae (rho $>0.25$ and $p<0.05$ ); FDP was significantly negatively correlated with Prevotella and Prevotellaceae (rho $<-0.25$ and $p<0.05$ ); LDL-C was significantly negatively correlated with Desulfovibrio (rho $<-0.25$ and $\mathrm{P}<0.05$ ); type 2 diabetes was significantly positively correlated with Lactobacillus and L. hamsteri (rho $>0.25$ and $p<0.05$ ), and significantly negatively correlated with Erysipelotrichaceae (rho $<-0.25$ and $p<0.05$ ); and $\mathrm{HbA} 1 \mathrm{c}$ was significantly positively correlated with $L$. hamsteri (rho $>0.25$ and $p<0.05$ ). Twelve taxa such as Streptococcus, Prevotella and Bacteroides were significantly different in NCA vs. SCAD, SCAD vs. AMI and NCA vs. AMI. In addition, according to the Gini coefficients of clinical indexes, we found that AST and LDL-C were highly significant for CAD grouping (Figure S2). Together, these data show that some differentially abundant taxa are significantly related to some key clinical indexes such as LDL-C, AST and $\mathrm{HbA1c}$, which provided a good theoretical basis for these differential taxa as biomarkers in future, and pending replication in later researches.

\subsection{Correlation analysis between clinical indexes and three types of metabolites}

To explore and compare the relationships between clinical indexes and the metabolites in serum, urine and faeces, we again performed a spearman correlation analysis between the three types of metabolites and clinical indexes (Figure 4). In the analysis of the correlation between faecal metabolites and clinical indexes, we found that FT3 was significantly positively correlated with hypoxanthine (rho $>0.25$ and $p$ $<0.05)$. In the correlation analysis of serum metabolites and clinical indexes, CRP was significantly negatively correlated with alanine and citrate (rho $<-0.25$ and $p<0.05)$; AST was significantly positively correlated with lactate (rho $>0.25$ and $p<0.05$ ), and negatively correlated with glycine (rho $<-0.25$ and $p$ 
$<0.05)$. Type 2 diabetes, blood glucose and $\mathrm{HbA} 1 \mathrm{c}$ was significantly negatively correlated with most metabolites (rho $<-0.25$ and $p<0.05)$ (Figure 4A).

In the correlation analysis between urine metabolites and clinical indexes, CRP was significantly positively correlated with taurine ( $r$ ho $>0.25$ and $p<0.05$ ), and significantly negatively correlated with citrate, hippurate. (rho $<-0.25$ and $p<0.05$ ); AST was significantly positively correlated with metabolites such as urea and galactose (rho $>0.25$ and $p<0.05$ ); FT3 was significantly negatively correlated with taurine (rho $<-0.25$ and $p<0.05$ ) and significantly positively correlated with creatinine, hippurate, glycolate and choline (rho $>0.25$ and $p<0.05$ ) (Figure 4B).

When comparing the results of the correlation analysis between the three types of metabolites and clinical indexes, we found that more metabolites in serum and urine were correlated with more clinical indexes than the faecal metabolome, this may indicate the serum and urine metabolome were more closely linked to the pathogenesis of CAD. It is worth noting that both type 2 diabetes vs. methanol and blood glucose vs. methanol showed significant negative correlation not only in the correlation analysis between serum metabolome and clinical indexes, but also in the correlation analysis between urine metabolome and clinical indexes. This may imply methanol may be correlated with both CAD and type 2 diabetes.

\subsection{Correlation analysis between gut microbiota and multi- type metabolites}

To reveal and compare the relationships between the gut microbiota and the three CAD subgroups of faecal, serum and urine metabolites. In our spearman correlation analysis (Figure 5), we found that in the correlation analysis between the faecal metabolome and gut microbiota (Figure 5A), Desulfovibrio was significantly positively correlated with sarcosine, dimethylamine, isovalerate (rho $>0.3$ and $p<0.05$ ); Consistent with a previous study[31], Faecalibacterium were significantly positively related to butyrate (rho $>0.3$ and $p<0.05$ ). We also found that Prevotella and Prevotellaceae were significantly positively correlated with hypoxanthine and xanthine (rho $>0.3$ and $p<0.05$ ); A.finegoldii, Alistipes and Rikenellaceae were significantly positively correlated with $\mathrm{p}$-cresol (rho $>0.3$ and $p<0.05$ ), which was consistent with the previous report[31].

When analyzing the correlation between the serum metabolome and gut microbiota (Figure 5B), Prevotella and Prevotellaceae showed significantly positive correlations with isoleucine and propyleneglycol (rho $>0.3$ and $p<0.05$ ); Lactobacillus and Lactobacillaceae showed significantly positive correlations with mannose (rho $>0.3$ and $p<0.05$ ); Lactobacillaceae was significantly negatively correlated with alanine (rho $<-0.3$ and $p<0.05$ ); C.aldenense was significantly positively correlated with lactate (rho $>0.3$ and $p<0.05$ ).

In the analysis of the correlation between urine metabolome and gut microbiota (Figure 5C), Prevotella and Prevotellaceae were significantly positively related to hippurate (rho $>0.3$ and $p<0.05$ ); M.fermentans 
and S.termitidis were significantly positively related to hypoxanthine and N-phenylacetylglycine, respectively (rho $>0.3$ and $p<0.05$ ); S.variabile was significantly positively correlated with galactose and urea (rho $>0.3$ and $p<0.05$ ).

Finally, when analyzing the correlation between the three types of metabolome and the gut microbiota, we found that $70 \%$ of the taxa in which correlated with the urine metabolites (rho $>0.3$ and $p<0.05$ ) were also correlated with the faecal metabolites. With the exception of Prevotella and Prevotellaceae, the gut microbes associated with the serum metabolome were different from those associated with the other two metabolomes. In addition, Prevotella and Prevotellaceae which were related to the three metabolomes were differentially abundant in SCAD vs. AMI.

\section{Discussion}

The study has explored the relationships among the gut microbiota, three types of metabolome and clinical indexes in an attempt to find potential CAD biomarkers; the biological roles of our identified markers (such as the genus Streptococcus and Prevotella, the metabolite citrate and creatinine) now warrant further investigation. In support of these findings, Liu et al. found that Prevotella was a potential therapeutic target for patients with cardiovascular disease[32]. It has been proved that the citrate cycle enhances cardioprotection[33]. And creatinine has been proposed to be a suitable biomarker for diabetes, $C A D$ and renal function[34]. Furthermore, we constructed relationship networks for the microbiota, metabolic pathways, and metabolites to try to explore CAD pathogenesis. We used the faecal metabolites to build the network because the metabolites detected in the faeces are more likely to come from intestinal microorganisms. Through network analysis, we delineated how these metabolites are related to the differentially abundant microbes. For example, we speculated that Escherichia releases alanine through the L-alanine biosynthesis pathway.

In the correlation analysis, serum/urine metabolites correlated more with clinical indexes than faecal; this finding constitutes a good option for researchers who want to further study the relationships between metabolites and clinical indexes. Compared with the serum metabolome, more correlations have been found between the faecal/urine metabolome and gut microbiota. By comparing the performance of the three metabolomes, we found that faeces/urine metabolites have stronger correlations with gut microbiota than serum metabolites.

190 faecal samples were sequenced by 16S rRNA sequencing, 60 faecal samples of which were also sequenced by metagenome sequencing. Compared with metagenomics sequencing, $16 \mathrm{~S}$ rRNA sequencing is not expensive and suitable for large cohort analysis. But it is difficult to detect the abundance of microbiotas in species level of $16 \mathrm{~S}$ data for technical reason. A similar situation occurs in the metabolome using NMR methods. Although metabolome sequencing based on NMR is highly specific, fewer metabolites can be detected. With the development of next generation sequencing (NGS) sequencing technology, metagenomic shotgun sequencing will become the mainstream, which will 
promote the further study of CAD in the future. Simultaneously, a stable untargeted metabolomics sequencing technology with high specificity, sensitivity and resolution need to be developed.

\section{Conclusions}

In our research, we used metagenome and multi-type metabolome profiling based on hundreds of samples to figure out the correlations among metabolites, microbiota and clinical indexes. We found serum and urine metabolites have stronger correlations with clinical indexes than faecal metabolites. Faecal and urine metabolites have stronger correlations with gut microbiota than serum metabolites. In addition, we firstly constructed the relationship networks of microbiota, metabolites and pathways. We believe our findings can help the researchers to further understand the pathogenesis of CAD.

\section{Methods}

\subsection{Participants and study design}

This study was approved by the Ethics Committee of Fuwai Hospital, Chinese Academy of Medical Sciences and Peking Union Medical College (Beijing, China) in accordance with the ethical standards of the 1964 Declaration of Helsinki and its later amendments. Written informed consent was obtained from all participants.

This is a single-center cross sectional study. We continuously recruited 190 participants under 65 years old with complete information on medical history, clinical and biochemical parameters from Fuwai Hospital, National Center for Cardiovascular Diseases of China. The diagnosis was made on the basis of symptoms, laboratory tests, ECG and coronary angiographic results. 190 participants including normal coronary artery (NCA group, $n=49$ ), stable coronary artery disease with the coronary artery stenosis $\geq$ $70 \%$ (sCAD group, $n=93$ ) and acute myocardial infarction (AMI group, $n=48$ ) were recruited between December 2016 and February 2017. The criteria for AMI included: 1) symptoms of chest pain at rest (> 20 min); 2) ischemic electrocardiographic changes: ST-segment changes and/or T-wave inversions; 3 ) significant increases in myocardial enzyme levels. For SCAD, the criteria included: 1) chest pain symptoms (< $10 \mathrm{~min}$ ) and electrocardiographic changes only after activity; 2) normal myocardial enzyme level. The coronary angiography was performed on all patients. Plaques or stenosis was not found in age- and sex-matched control subjects. All enrolled participants in the NCA, SCAD and AMI group who were suspected of CAD underwent CAG and had no history of unstable angina, myocardial infarction, stroke, cancers, or coronary revascularization. The angiographic data were confirmed independently by two observers in this study.

\subsection{Nuclear Magnetic Resonance (NMR) Sample collection and preparation}


Serum $\llbracket$ before the coronary angiography surgery $\llbracket$ and urine $\$ early morning urinary $\llbracket$ samples were collected and centrifuged at $278 \mathrm{~K}$ at $3,000 \mathrm{~g}$ for $10 \mathrm{~min}$, the supernatants of samples were stored at $-80^{\circ} \mathrm{C}$ for metabolic profile establishment and statistical analysis. Faeces samples were stored at $-80^{\circ} \mathrm{C}$ after homogenate with phosphate buffer (0.2 M NaH2PO4/K2HPO4, pH 7.4). Samples were prepared using the previously reported method[16].

\subsection{NMR Spectra Acquisition and Processing}

All NMR spectra were recorded at $298 \mathrm{~K}$ using a Bruker Avance $500 \mathrm{MHz}$ spectrometer $(1 \mathrm{H}$ frequency: $500.13 \mathrm{MHz}$; Bruker, Germany). For quantitative metabolomics profiling of filtered serum, urine, and faeces, spectra were processed with the Chenomx NMR Suite 7.5 software (Chenomx Inc., Edmonton, Canada) using the "targeted profiling" approach[17]. Open database sources, including the KEGG, MetaboAnalyst, Human Metabolome Database, and METLIN, were used to identify metabolic pathways[18, 19].

\subsection{NMR Multivariate Data Analysis}

Output data were processed with the SIMCA-P+ 14.0 software (Umetrics, Sweden) to elucidate patterns in metabolite concentration shifts. Statistical analysis was also conducted with SPSS19.0 (IBM; USA) using the two-tailed Student's t-test. P-value of less than 0.05 was considered to be statistically significant between two groups.

\subsection{Human faecal sample collection and DNA extraction}

Fresh faeces samples were collected from 190 subjects, and then delivered from Fuwai Hospital to the laboratory in an ice bag using insulating polystyrene foam containers. DNA was extracted using an EZNA $^{\text {TM }}$ stool DNA isolation kit (Omega Bio-Tek, VWR, Herlev, Denmark). The DNA was then eluted in $50 \mu \mathrm{L}$ of elution buffer and stored at $-80^{\circ} \mathrm{C}$.

\subsection{DNA library construction and sequencing}

DNA library was constructed using the TruSeq Nano DNA LT Library Preparation Kit (FC-121-4001, Illumina, San Diego, CA, USA). The resulting libraries were sequenced on an Illumina HiSeq 4000 sequencer (Illumina, San Diego, CA, USA). The running mode of metagenomics was paired-end of $150 \mathrm{bp}$ and the running mode of $16 \mathrm{~S}$ rRNA sequencing was paired-end of $300 \mathrm{bp}$.

\subsection{Sequencing data analysis}


QIIME 2 was used to process 16 S rRNA sequencing data (Figure S1). Sequence quality control, feature table construction and filter chimeric sequences were performed by DADA2 plugins[20]. Features were created by clustering sequences with $100 \%$ similarity. Representative sequences for each feature were used to construct a rooted phylogenetic tree by q2-phylogeny plugin. The script will randomly subsample the counts from each sample to the 7327 sequences. Alpha and beta diversity were generated by q2diversity plugin. Shannon's index, the observed OTUs, and evenness were evaluated. A normalized feature abundance table was used for the constrained principal coordinate analysis (cPCoA). Taxonomic analysis was performed by q2-feature-classifier plugin. Differential abundance taxa were generated by LEfSe (LDA>2)[21].

Quality control for the metagenomics shotgun sequencing data was conducted using FastQC (http://www.bioinformatics.babraham.ac.uk/projects/fastqc/). Low quality reads and adapter sequences were removed by Trimmomatic[22]. Taxonomic profiles were generated using MetaPhIAn v2.662[23] and pathways enrichment was done by HUMAnN2[24]. The interactions of pathways and metabolites were integrated using MetaCyc and the relationship networks contained microbiota, metabolites and pathways were visualized by Cytoscape[25, 26].

\subsection{Statistical analyses}

The Gini coefficients of clinical indexes were generated using R scripts. The wilcox and Fisher's test were used to analyze the differential clinical indexes for continuous and categorical variables, respectively. Spearman correlations between microbiota, metabolites and clinical indexes were calculated using $\mathrm{R}$ scripts. The visual presentation of multiple omics correlations was performed using the pheatmap package in $\mathrm{R}$.

\subsection{Data availability}

The datasets are available in the repository of the Genome Sequence Archive Sequence Database of National Genomics Data Center (https://bigd.big.ac.cn/) under the accession number CRA002142.

\section{List Of Abbreviations}




\begin{tabular}{|c|c|}
\hline CAD & coronary artery disease \\
\hline 16S rRNA-seq & 16S rRNA sequencing \\
\hline STEMI & ST-segment elevation myocardial infarction \\
\hline post-Ml & post myocardial infarction \\
\hline WGS & whole genome sequencing \\
\hline NCA & normal coronary artery \\
\hline sCAD & stable coronary artery disease \\
\hline AMI & acute myocardial infarction \\
\hline NMR & nuclear magnetic resonance \\
\hline DSS & dimethyl-silapentane-sulfonate \\
\hline KEGG & kyoto encyclopedia of genes and genomes \\
\hline PCA & principal component analysis \\
\hline PLS-DA & partial least squares discriminant analysis \\
\hline PCR & polymerase chain reaction \\
\hline rRNA & ribosomal RNA \\
\hline OTU & operational taxonomic unit \\
\hline cPCoA & constrained principal coordinate analysis \\
\hline ECG & electrocardiograms \\
\hline PN & percentage of neutrophils \\
\hline ANC & absolute neutrophil count \\
\hline PL & percentage of lymphocyte \\
\hline ALC & absolute lymphocyte count \\
\hline PM & percentage of monocyte \\
\hline AMC & absolute monocyte count \\
\hline RBC & red blood cell \\
\hline PLT & platelet \\
\hline FDP & fibrin degradation products \\
\hline BMI & body mass index \\
\hline TC & total cholesterol \\
\hline
\end{tabular}

Page 20/29 


\begin{tabular}{|ll|}
\hline LDL-C & high density lipoprotein \\
\hline CRP & C-reactive protein \\
WBC & white blood cell \\
ALT & alanine aminotransferase \\
\hline AST & aspartate aminotransferase \\
CK-MB & creatine kinase myocardic isoenzyme \\
CK & creatine kinase \\
\hline LDH & lactate dehydrogenase \\
\hline TSH & thyroid-stimulating hormone \\
\hline CTn & cardiac troponin \\
\hline URL & upper reference limit \\
\hline
\end{tabular}

\section{Declarations}

\section{Ethics approval and consent to participate}

This study was approved by the Ethics Committee of Fuwai Hospital, Chinese Academy of Medical Sciences and Peking Union Medical College (Beijing, China) in accordance with the ethical standards of the 1964 Declaration of Helsinki and its later amendments. Written informed consent was obtained from all participants.

\section{Consent for publication}

The authors consent for publication

\section{Availability of data and materials}

The datasets generated and/or analyzed during the current study are available in the repository of the Genome Sequence Archive Sequence Database of National Genomics Data Center (https://bigd.big.ac.cn/) under the accession number CRA002142.

\section{Competing interests}

The authors declare that they have no competing interests. 


\section{Funding}

This work was supported by Chinese Academy of Medical Sciences Innovation Fund for Medical Sciences [CIFMS 2016-I2M-1-009], Disciplines Construction Project [201920200807], Fundamental Research Funds for the Central Universities [3332019068], The Drug Innovation Major Project [2018ZX09711001-003-011], National Program on Key Basic Research Project of China [973 Program, No. 2015CB554405].

\section{Authors' contributions}

$\mathrm{HZ}$, SH and YT conceived the research and designed the experiments. YT recruited patients, collected clinical samples and registered clinical information. YW, CD, FG, SS and QL performed the experiments. $\mathrm{CD}, \mathrm{ZH}, \mathrm{QZ}$ and $\mathrm{KL}$ analyzed the data. $\mathrm{HZ}, \mathrm{CD}$ and $\mathrm{ZH}$ wrote the manuscript. JL revised the manuscript. $H Z$, JL and $Y T$ offered funds for the research.

\section{Acknowledgements}

We thank all study participants, research staffs, and students who participated in this work.

\section{References}

1. Khera AV, Kathiresan S. Genetics of coronary artery disease: discovery, biology and clinical translation. Nature reviews Genetics. 2017;18(6):331-44.

2. Piepoli MF, Hoes AW, Agewall S, Albus C, Brotons C, Catapano AL, Cooney MT, Corra U, Cosyns B, Deaton C, et al. 2016 European Guidelines on cardiovascular disease prevention in clinical practice. Revista espanola de cardiologia. 2016;69(10):939.

3. Ford TJ, Corcoran D, Berry C. Stable coronary syndromes: pathophysiology, diagnostic advances and therapeutic need. Heart. 2018;104(4):284-92.

4. Alpert JS. The Fourth Edition of the Universal Definition of Myocardial Infarction. Am J Med. 2018;131(11):1265-6.

5. Gorenoi V, Schonermark MP, Hagen A. CT coronary angiography vs. invasive coronary angiography in CHD. GMS Health Technol Assess. 2012;8:Doc02.

6. Liu H, Chen X, Hu X, Niu H, Tian R, Wang H, Pang H, Jiang L, Qiu B, Chen X, et al: Alterations in the gut microbiome and metabolism with coronary artery disease severity. Microbiome 2019, 7(1).

7. Qin J, Li Y, Cai Z, Li S, Zhu J, Zhang F, Liang S, Zhang W, Guan Y, Shen D, et al. A metagenome-wide association study of gut microbiota in type 2 diabetes. Nature. 2012;490(7418):55-60.

8. Yu J, Feng Q, Wong SH, Zhang D, Liang QY, Qin Y, Tang L, Zhao H, Stenvang J, Li Y, et al. Metagenomic analysis of faecal microbiome as a tool towards targeted non-invasive biomarkers for 
colorectal cancer. Gut. 2017;66(1):70-8.

9. Zhang X, Zhang D, Jia H, Feng Q, Wang D, Liang D, Wu X, Li J, Tang L, Li Y, et al. The oral and gut microbiomes are perturbed in rheumatoid arthritis and partly normalized after treatment. Nature medicine. 2015;21(8):895-905.

10. Ussar S, Griffin NW, Bezy O, Fujisaka S, Vienberg S, Softic S, Deng L, Bry L, Gordon JI, Kahn CR. Interactions between Gut Microbiota, Host Genetics and Diet Modulate the Predisposition to Obesity and Metabolic Syndrome. Cell Metabol. 2015;22(3):516-30.

11. Zhong H, Penders J, Shi Z, Ren H, Cai K, Fang C, Ding Q, Thijs C, Blaak EE, Stehouwer CDA, et al. Impact of early events and lifestyle on the gut microbiota and metabolic phenotypes in young school-age children. Microbiome. 2019;7(1):2.

12. Aaron C, Ericsson SA, Marina M, Hanson SB, Busi TW, Parker RJ, Schehr MA, Hankins CE, Ahner, Justin W, Davis. Craig L Franklin, James M Amos-Landgraf, Elizabeth C Bryda: Differential susceptibility to colorectal cancer due to naturally occurring gut microbiota. Oncotarget $2015,6(32)$.

13. Jie Z, Xia H, Zhong SL, Feng Q, Li S, Liang S, Zhong H, Liu Z, Gao Y, Zhao H, et al. The gut microbiome in atherosclerotic cardiovascular disease. Nat Commun. 2017;8(1):845.

14. Li J, Zhao F, Wang Y, Chen J, Tao J, Tian G, Wu S, Liu W, Cui Q, Geng B, et al. Gut microbiota dysbiosis contributes to the development of hypertension. Microbiome. 2017;5(1):14.

15. Zhou X, Li J, Guo J, Geng B, Ji W, Zhao Q, Li J, Liu X, Liu J, Guo Z, et al. Gut-dependent microbial translocation induces inflammation and cardiovascular events after ST-elevation myocardial infarction. Microbiome. 2018;6(1):66.

16. Jiang CY, Yang KM, Yang L, Miao ZX, Wang YH, Zhu HB. A (1)H NMR-Based Metabonomic Investigation of Time-Related Metabolic Trajectories of the Plasma, Urine and Liver Extracts of Hyperlipidemic Hamsters. PloS one. 2013;8(6):e66786.

17. Weljie AM, Newton J, Mercier P, Carlson E, Slupsky CM. Targeted profiling: quantitative analysis of $1 \mathrm{H}$ NMR metabolomics data. Analytical chemistry. 2006;78(13):4430-42.

18. Kanehisa M, Goto S. KEGG: kyoto encyclopedia of genes and genomes. Nucleic acids research. 2000;28(1):27-30.

19. Wishart DS, Feunang YD, Marcu A, Guo AC, Liang K, Vazquez-Fresno R, Sajed T, Johnson D, Li C, Karu N, et al: HMDB 4.0: the human metabolome database for 2018. Nucleic acids research 2018, 46(D1):D608-D617.

20. Bolyen E, Rideout JR, Dillon MR, Bokulich NA, Abnet CC, Al-Ghalith GA, Alexander H, Alm EJ, Arumugam M, Asnicar F, et al. Reproducible, interactive, scalable and extensible microbiome data science using QIIME 2. Nature biotechnology. 2019;37(8):852-7.

21. Segata N, Izard J, Waldron L, Gevers D, Miropolsky L, Garrett WS, Huttenhower C. Metagenomic biomarker discovery and explanation. Genome Biol. 2011;12(6):R60.

22. Bolger AM, Lohse M, Usadel B. Trimmomatic: a flexible trimmer for Illumina sequence data. Bioinformatics. 2014;30(15):2114-20. 
23. Segata N, Waldron L, Ballarini A, Narasimhan V, Jousson O, Huttenhower C. Metagenomic microbial community profiling using unique clade-specific marker genes. Nature methods. 2012;9(8):811-4.

24. Franzosa EA, Mclver LJ, Rahnavard G, Thompson LR, Schirmer M, Weingart G, Lipson KS, Knight R, Caporaso JG, Segata N, et al. Species-level functional profiling of metagenomes and metatranscriptomes. Nat Methods. 2018;15(11):962-8.

25. Caspi R, Billington R, Keseler IM, Kothari A, Krummenacker M, Midford PE, Ong WK, Paley S, Subhraveti P, Karp PD: The MetaCyc database of metabolic pathways and enzymes-a 2019 update. Nucleic acids research 2019.

26. Shannon P, Markiel A, Ozier O, Baliga NS, Wang JT, Ramage D, Amin N, Schwikowski B, Ideker T. Cytoscape: a software environment for integrated models of biomolecular interaction networks. Genome research. 2003;13(11):2498-504.

27. Ndrepepa G, Holdenrieder S, Colleran R, Cassese S, Xhepa E, Fusaro M, Laugwitz KL, Schunkert H, Kastrati A. Inverse association of alanine aminotransferase within normal range with prognosis in patients with coronary artery disease. Clin Chim Acta. 2019;496:55-61.

28. Dhar I, Lysne V, Seifert R, Svingen GFT, Ueland PM, Nygard OK. Plasma methionine and risk of acute myocardial infarction: Effect modification by established risk factors. Atherosclerosis. 2018;272:175-81.

29. Gu H, Chen W, Yin J, Chen S, Zhang J, Gong J. Methionine sulfoxide reductase A rs10903323 G/A polymorphism is associated with increased risk of coronary artery disease in a Chinese population. Clinical biochemistry. 2013;46(16-17):1668-72.

30. Investigators T, Alexander JH, Reynolds HR, Stebbins AL, Dzavik V, Harrington RA, Van de Werf F, Hochman JS. Effect of tilarginine acetate in patients with acute myocardial infarction and cardiogenic shock: the TRIUMPH randomized controlled trial. JAMA. 2007;297(15):1657-66.

31. Yi Wan FW, Yuan J, Li J, Jiang D, Zhang J, Li H, Wang R, Tang J, Huang T, Zheng J, Sinclair AJ. Jim Mann, Duo Li: Effects of dietary fat on gut microbiota and faecal metabolites, and their relationship with cardiometabolic risk factors: a 6-month randomised controlled-feeding trial. Gut 2019.

32. Liu Z, Li J, Liu H, Tang Y, Zhan Q, Lai W, Ao L, Meng X, Ren H, Xu D, et al. The intestinal microbiota associated with cardiac valve calcification differs from that of coronary artery disease. Atherosclerosis. 2019;284:121-8.

33. Lu H, Chen Y, Li L. Metabolic Pathway Genes Associated with Susceptibility Genes to Coronary Artery Disease. International journal of genomics. 2018;2018:9025841.

34. Bagheri B, Radmard N, Faghani-Makrani A, Rasouli M. Serum Creatinine and Occurrence and Severity of Coronary Artery Disease. Medical archives. 2019;73(3):154-6.

\section{Figures}




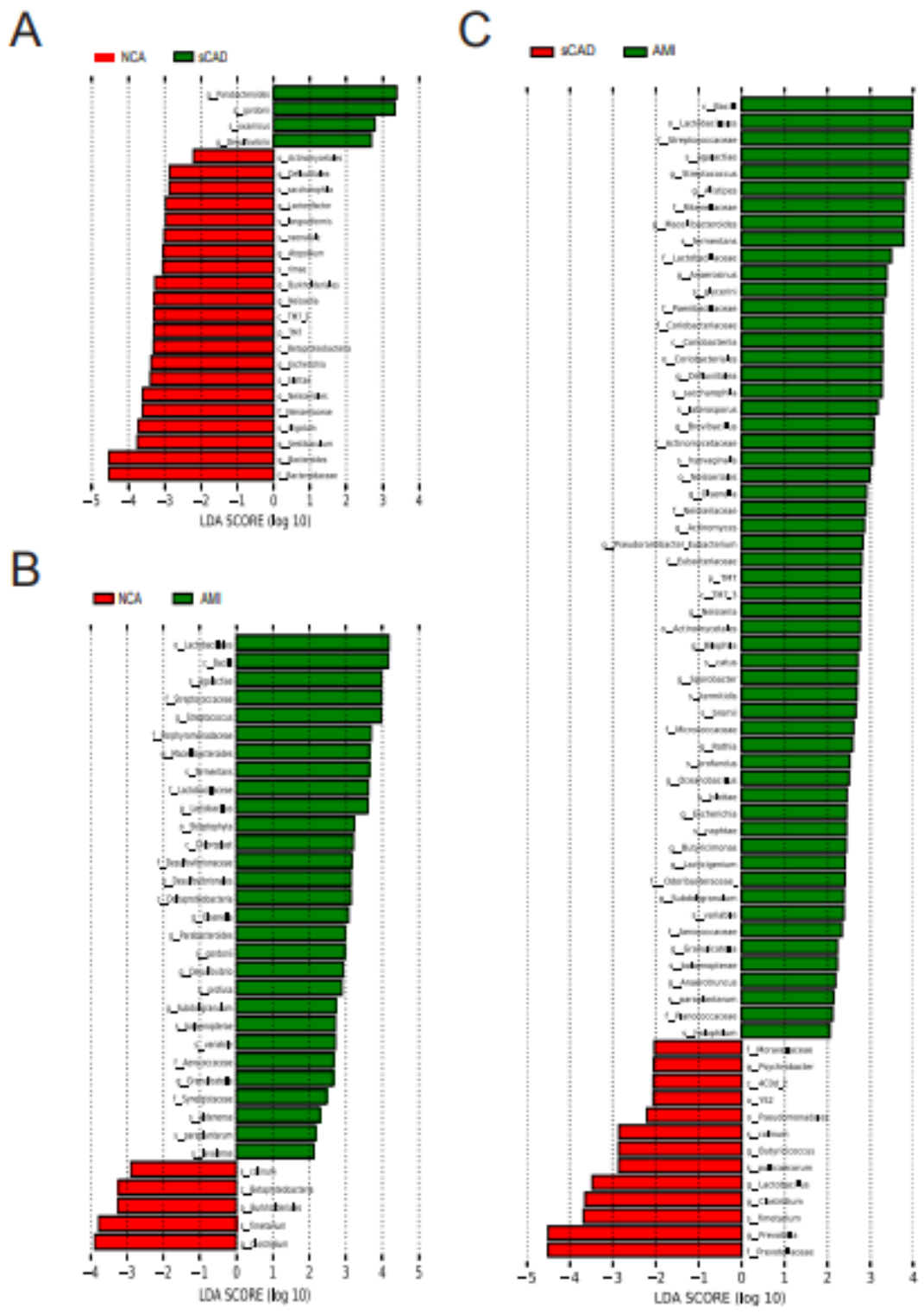

Figure 1

Identification of the enriched bacteria associated with CAD subgroups using LEfSe analysis LEfSe plots showed the most differentially abundant taxa. Only taxa meeting LDA scores $>2$ are shown. A: Taxa enriched for NCA in red; taxa enriched for SCAD in green. B: Taxa enriched for SCAD in red; taxa enriched for AMI in green. C: Taxa enriched for NCA in red; taxa enriched for AMI in green. 
A

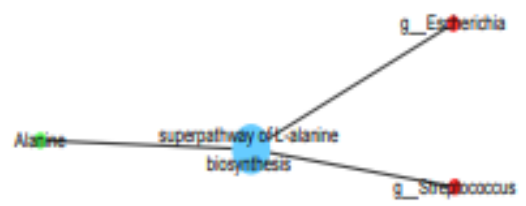

B

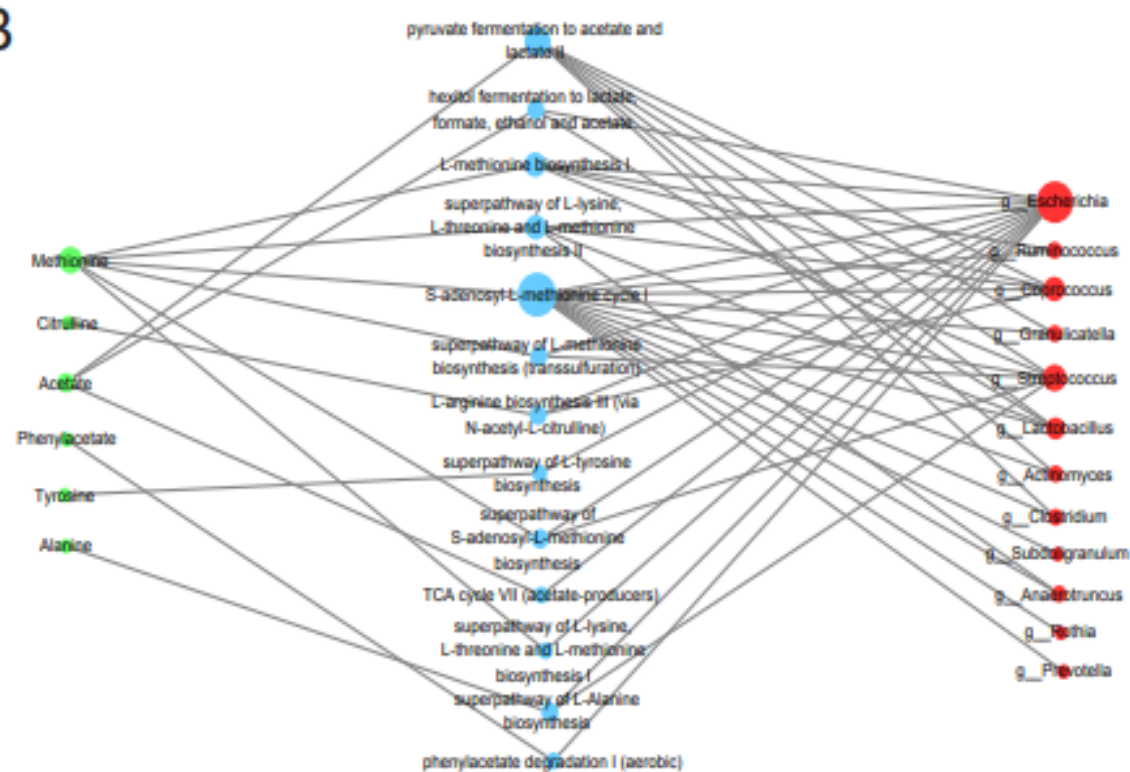

C

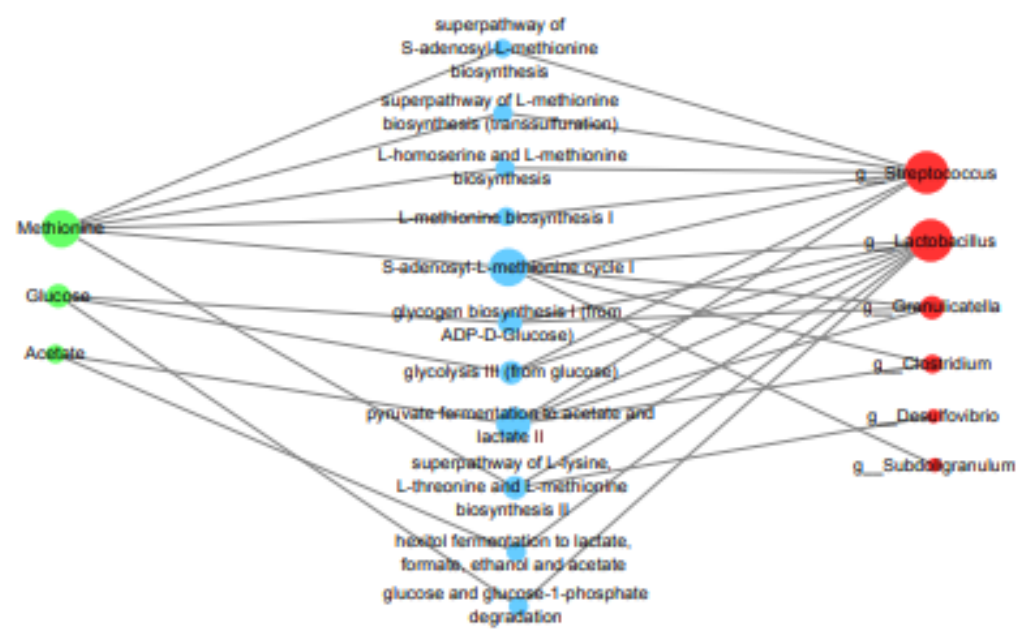

Figure 2

Integrated network of microbiota, metabolites and metabolic pathways among CAD subgroups The direction of enrichment was determined by MetaCyc and HUMAnN2. Sizes of the nodes were in proportion with numbers of links. Green, red and blue nodes represent metabolites, microbiota and related pathways, respectively. A: NCA vs SCAD; B: SCAD vs AMI; C: NCA vs AMI 


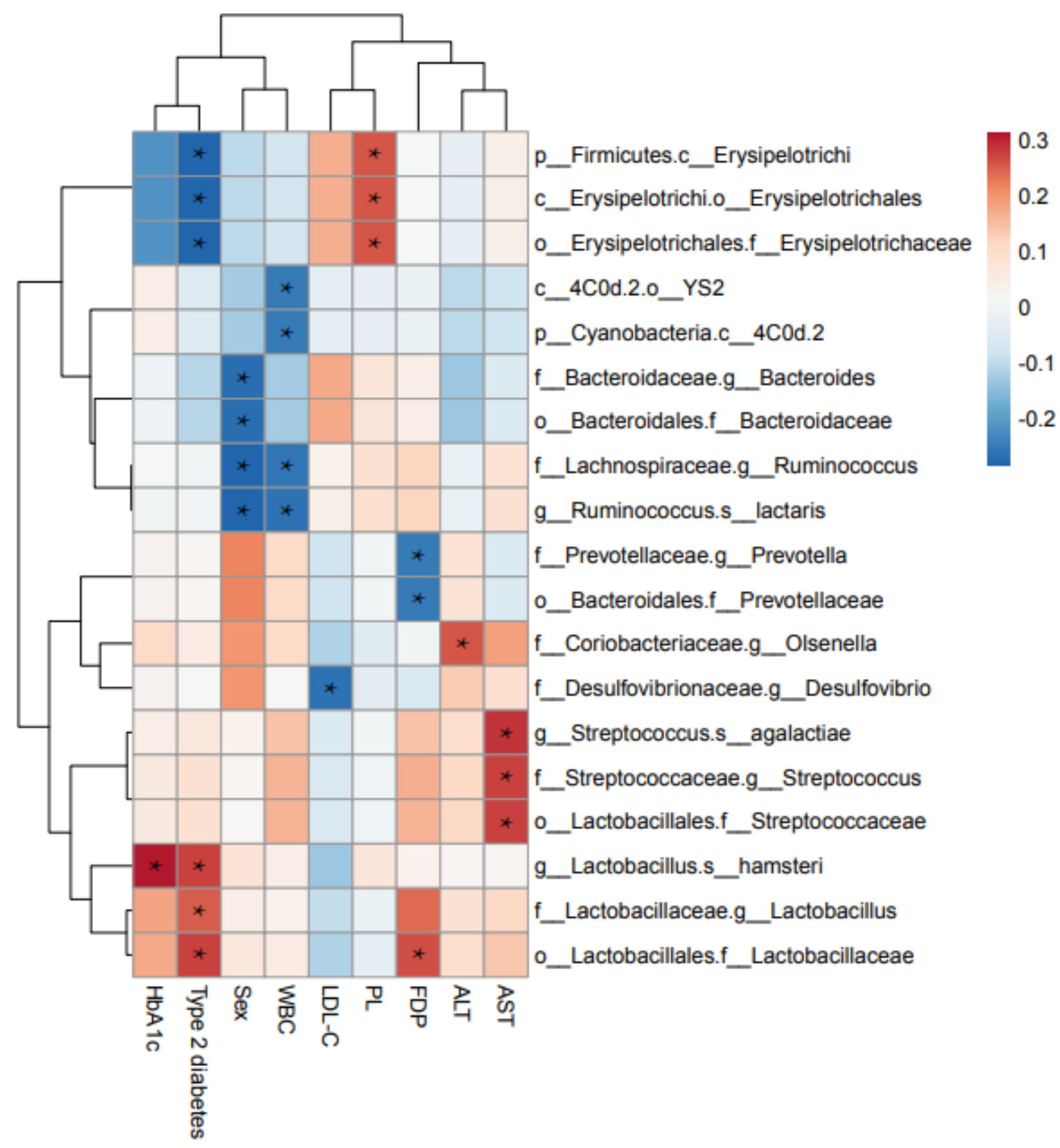

Figure 3

Heatmap of correlation analysis between clinical indexes and microbiota Spearman correlation analysis was performed to assess the correlation of clinical indexes with gut microbiota in the NCA, SCAD and AMI groups. ${ }^{*} p<0.05 \& \&$ rho $>0.25$ 
A

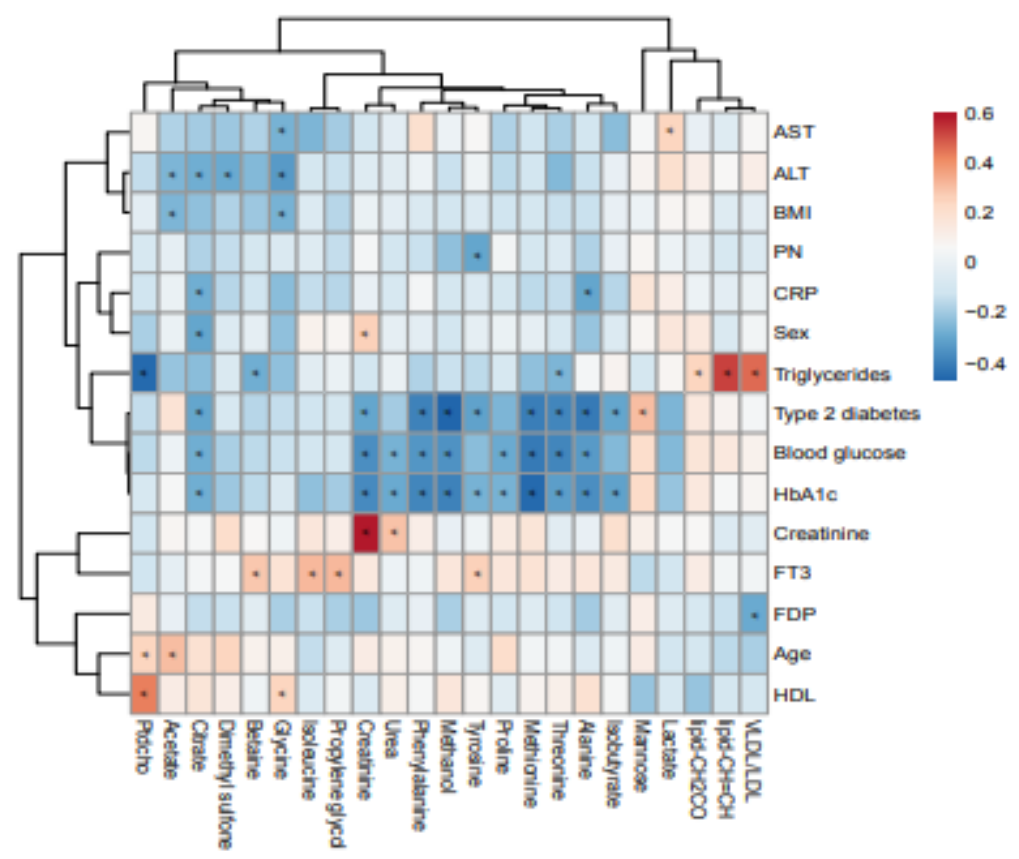

B

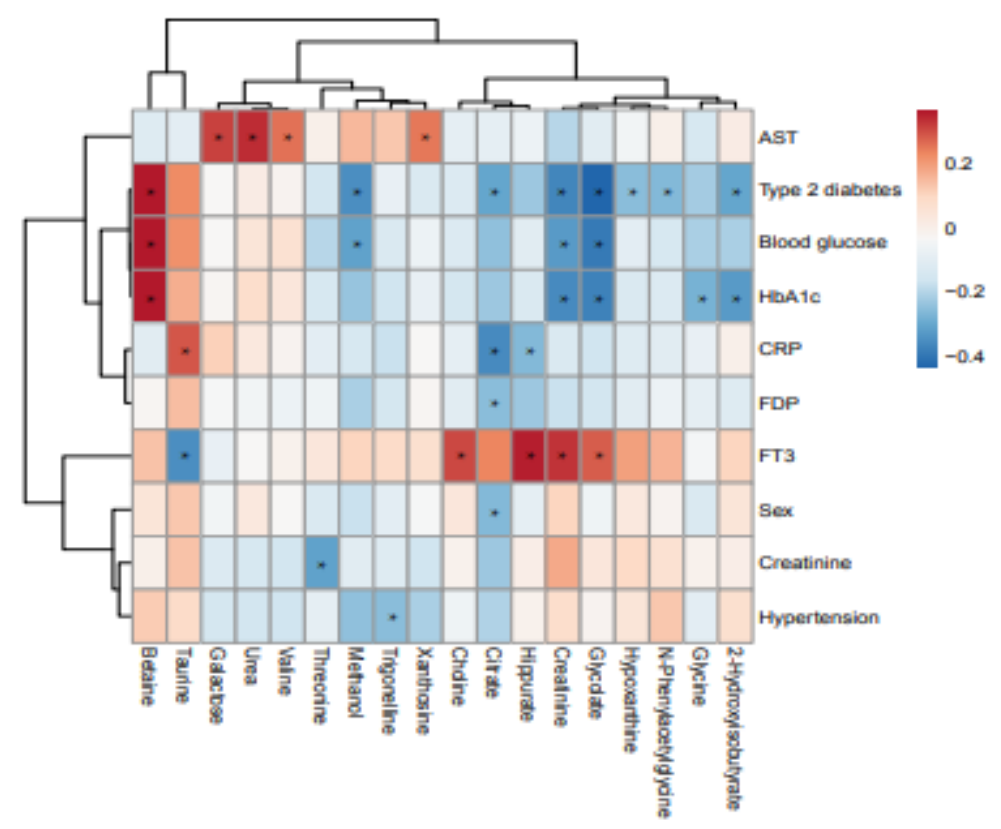

Figure 4

Heatmap of correlation analysis between clinical indexes and three types of metabolites Spearman correlation analysis was performed between serum metabolites and clinical indexes (Figure 4A), urine metabolites and clinical indexes (Figure 4B). Red panel indicated positive correlation, while blue panel suggested negative correlation. ${ }^{*} p<0.05 \& \&$ rho $>0.25$. 
A
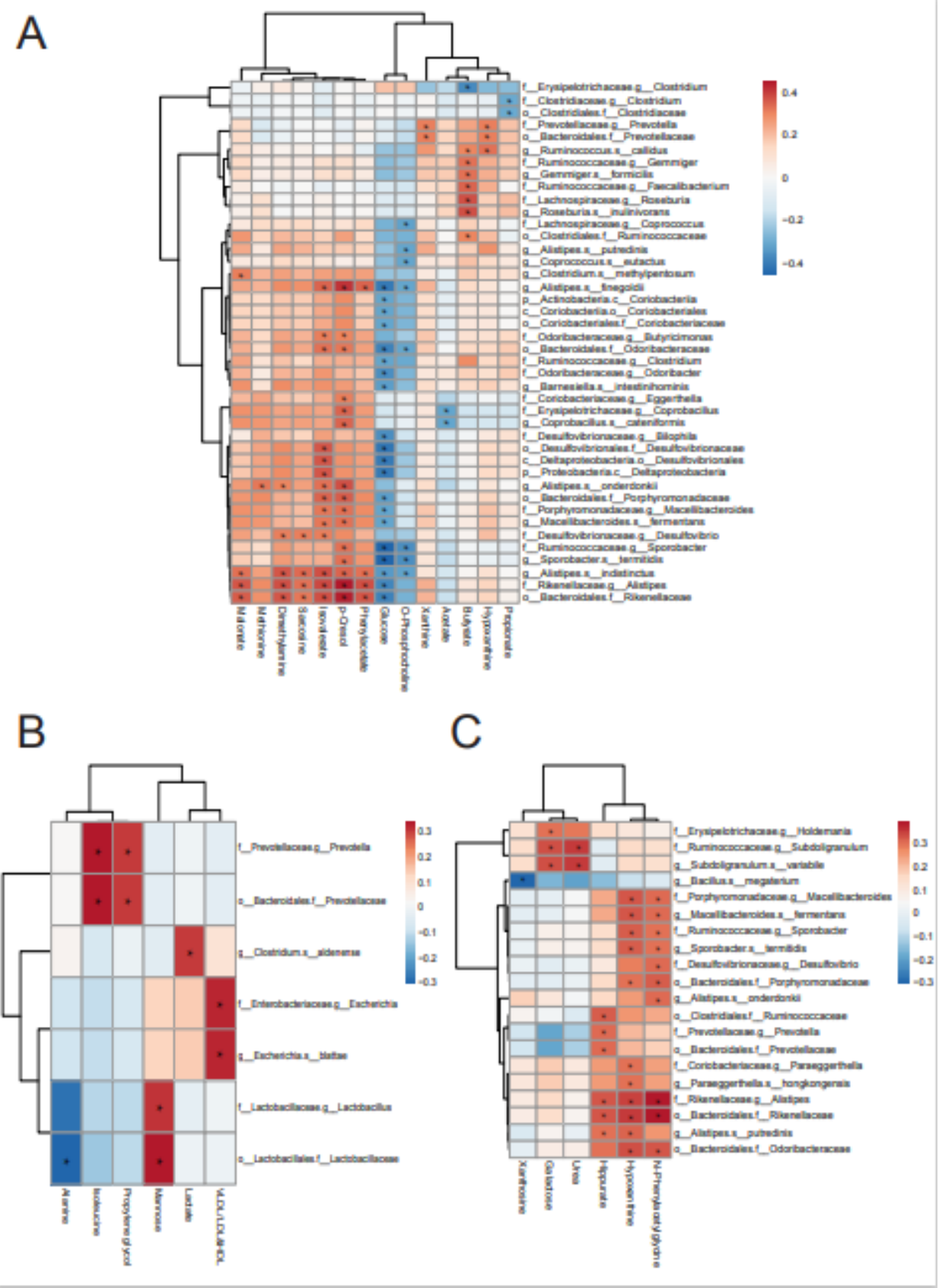

Figure 5

Heatmap of correlation analysis between microbiota and three types of metabolites Spearman's correlation coefficients between the abundance of microbiota and the level of faecal/serum/urine metabolic patterns (Figure 5). Red, negative correlation; blue, positive correlation. * $p<0.05 \& \&$ rho $>0.25$.

\section{Supplementary Files}

This is a list of supplementary files associated with this preprint. Click to download.

- SupplementalMaterial.docx 